\title{
COMERCIALIZACIÓN DE LA LECHE EN LA PROVINCIA DE CONCEPCIÓN, VALLE DEL MANTARO, JUNÍN - PERÚ
}

\author{
COMERCIALIZATION OF MILK IN THE PROVINCE OF CONCEPCIÓN, MANTARO \\ VALLEY, JUNÍN - PERU
}

${ }^{1}$ Cindybell Gamboa y ${ }^{2}$ Waldemar Mercado

\begin{abstract}
Resumen
El objetivo de estudio fue verificar las interrelaciones existentes entre los abastecedores de insumos, productores e intermediarios en el comercio de la leche para determinar tanto la red de comercialización como la rentabilidad asociada a cada actor de la cadena de la leche en el Valle del Mantaro, provincia de Concepción - Junín. El trabajo se ejecutó durante el periodo de seca (2011) y se recopiló la información mediante encuestas a 146 productores, 6 acopiadores rurales, 20 procesadores y se efectuaron 3 talleres participativos. Los resultados mostraron que el pequeño productor posee bajo nivel tecnológico dado la deficiente articulación en la provisión de bienes productivos y servicios técnicos, que repercuten en rendimientos inferiores al promedio y alto costo de producción unitario en relación al precio percibido por el bien. Además están inmersos en complejos canales de comercialización (doce en total identificados), y que son centralizados por las procesadoras (formales y artesanales), generando desigualdades en la distribución de márgenes netos unitarios en la red comercial. Se concluye que en el mercado rural el sistema de comercialización es ineficiente, pues coexisten simultáneamente mercados formales e informales que impactan en los productores e intermediarios, en tanto, los consumidores de mayor nivel económico son abastecidos por la industria formal, y los demandantes de menores ingresos son atendidos por los transformadores artesanales, debido principalmente a la falta de estandarización para la venta del producto y escaso control para el cumplimiento de las regulaciones existentes.
\end{abstract}

Palabras claves: cadena de suministro, comercialización, economía de la tierra, ganado vacuno, ganancia y producción agrícola.

\begin{abstract}
The aim of this study was to verify the interrelations between suppliers, producers and intermediaries in the trade of raw milk to determinate the network commercialization and also the profitability in each agents of milk chain in the Mantaro valley, province of Concepcion, Junín. The work was executed during the dry season (2011) and we collected information by surveys on 146 farmers, 6 rural collectors and 20 processing plants, and three participative workshops. The results showed that the small producer has low-tech process and it is deficient in providing productive assets and technical services, which affect yields below average and high unit cost of production in relation to the price charged for the good. Hence the small producers are involved in complex commercialization channels (more than twelve channels identified) and centralized by the processing plants (formals and artisanal), generating an inequality in the distribution of net margins per unit in the commercialization network. Finally we conclude that in the rural market the commercialization system of raw milk is inefficient because formal and informal markets that impact on producers and intermediaries decisions coexist simultaneously. Meanwhile, consumers with higher incomes are served by the formal industry, and consumers with lower incomes are served by artisanal processors, due mainly to the lack of standardization of the products for sales and to the little control for compliance of the existing regulations.
\end{abstract}

Key words: cattle, commercialization, land economics, profit, raw milk production and supply chain.

\section{Introducción}

Los mercados agrarios reflejan las peculiaridades de la producción agraria, atomizada, dispersa, estacional, de productos perecederos en mayor o menor grado y condicionada por factores naturales que da origen a la variabilidad de los precios y otorga a la comercialización un papel importante para atender la demanda (Caldentey y Gómez 1992, Cannock y Gonzáles-Zuñiga 1994, Mendoza 1987, Martínez 2005).
La comercialización agraria son todas las actividades realizadas para lograr el traslado de productos agrícolas, desde el punto de producción hasta que llega al consumidor final en los mercados internos y externos, siendo esenciales los procesos de concentración de la producción, distribución e igualación del producto (Mendoza 1987, Martínez 2005).

Mendoza (1987) clasifica el sistema de comercialización según la estructura funcional y el centro de gravitación de 
decisiones. Un sistema centralizado es donde el flujo del bien desde el productor hasta el consumidor sigue canales cuyas decisiones y funciones más importantes se realizan a nivel urbano. El sistema descentralizado desplaza el poder hacia el detallista y se realizan mayor número de funciones de comercialización a nivel rural, debido a la integración horizontal y a la capacidad de crear y mantener un mercado, siendo el contacto entre detallistas y productores más directo.

Los canales de comercialización son parte de una red que intermedia la transferencia del producto, representando la relación entre los agentes de intermediación y permitiendo conocer de manera sistemática el flujo o circulación de los productos entre su origen y destino (Coscia 1978, Meléndez 1984, Alarcón y Ordinola 2002). El conjunto de canales conforman la red de comercialización. Alarcón y Ordinola (2002) establecen una tipología de redes y canales de comercialización en base al grado de intermediación y de la estructura de las redes, que se presenta en la tabla 1.

Tabla 1. Tipología de redes y canales de comercialización.

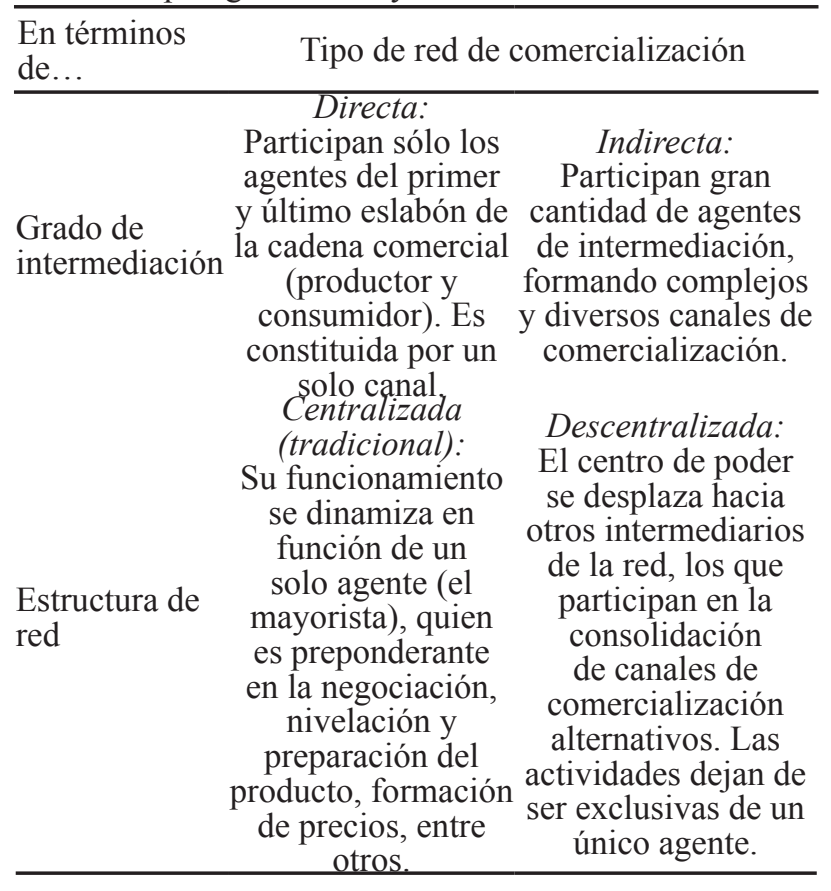

Fuente: Alarcón y Ordinola (2002), Elaboración propia.

Para determinar los márgenes de comercialización y la rentabilidad de las operaciones comerciales deben verificarse los costos de producción, los costos del mercadeo, los precios recibidos y pagados por los agentes.

Los costos de producción son la valoración económica de los recursos incurridos para la obtención de un bien en un periodo, y representan gastos en compra de insumos, mano de obra (contratada y familiar), costo de la tierra, depreciación, entre otros (Álvarez y Sánchez 1998). Los costos de comercialización, constituyen el valor de los insumos usados para agregar utilidad al producto. Los costos de mercadeo varían de un producto a otro, dependiendo de muchos factores, siendo estos gastos realizados por todos los agentes participantes en el mercadeo (Alarcón y Ordinola 2002).

El margen de comercialización es la diferencia entre el precio que paga el consumidor por un producto y el precio recibido por el cultivador (Mendoza 1987). Existe el margen bruto y el margen neto de comercialización. La diferencia conceptual entre ambos radica en la composición del costo absorbido por el comercializador.

La rentabilidad proporciona información sobre la utilidad neta de cada uno de los agentes involucrados como proporción de los costos incurridos. La rentabilidad neta por hectárea es el cociente del margen neto por hectárea con el costo total ${ }^{3}$ y sirve para determinar si el productor ganó o perdió dinero después de vender el producto, siendo útil para evaluar su eficiencia en la asignación de sus recursos y realizar comparaciones, siendo indispensable para la toma de decisiones de los productores, los intermediarios e incluso el Estado. En general, cuanto más larga y compleja es la red de comercialización de un producto, mayor serán sus costos de comercialización y menor el precio pagado al productor, pues el mercado homogeniza los precios en el punto de venta en forma independiente a la procedencia del producto, siempre y cuando no hayan mecanismos de diferenciación del producto o reconocimiento especial de parte de los consumidores.

En la agricultura familiar predominante en el Valle del Mantaro-Junín, las decisiones de producción se desenvuelven entre la lógica de economía campesina y la racionalidad empresarial de los transformadores, siendo que en el sistema de comercialización existente participan los proveedores de insumos productivos y servicios técnicos, los productores de leche, los transformadores y los comercializadores.

Por tanto, el objetivo del estudio fue verificar las interrelaciones existentes entre abastecedores de insumos, productores, transformadores e intermediarios en el comercio de la leche para determinar tanto la red de comercialización como la rentabilidad asociada a los productores y agentes participantes.

\section{Metodología}

El Valle del Mantaro se localiza sobre los $3270 \mathrm{msnm}$ en el departamento de Junín, políticamente incluye cuatro provincias: Huancayo, Concepción, Jauja, Chupaca y un total de 29 distritos. El valle es reconocido por su capacidad de abastecimiento de alimentos a la ciudad de Lima Metropolitana, principal mercado nacional.

El estudio se realizó en cuatro distritos (Concepción, Matahuasi, Mito y Orcotuna) de la provincia de Concepción que pertenecen al Valle del Mantaro (Fig. 1), ellos concentran la mayor producción agropecuaria en alfalfa que es $65 \%$ respecto al total provincial y $48 \%$ en leche fresca al que se dedican $40 \%$ de los productores 3Considerando los costos monetarios y los no monetarios que son generados en el proceso productivo. 
(Municipalidad Provincial de Concepción 2006). Los distritos son conectados con las ciudades de Jauja y Huancayo (Región Junín) y vía la carretera central con
Lima (capital del país) con pista de doble vía asfaltada y distancia de $277 \mathrm{Km}$. La distancia de Huancayo a Concepción es $21 \mathrm{Km}$.

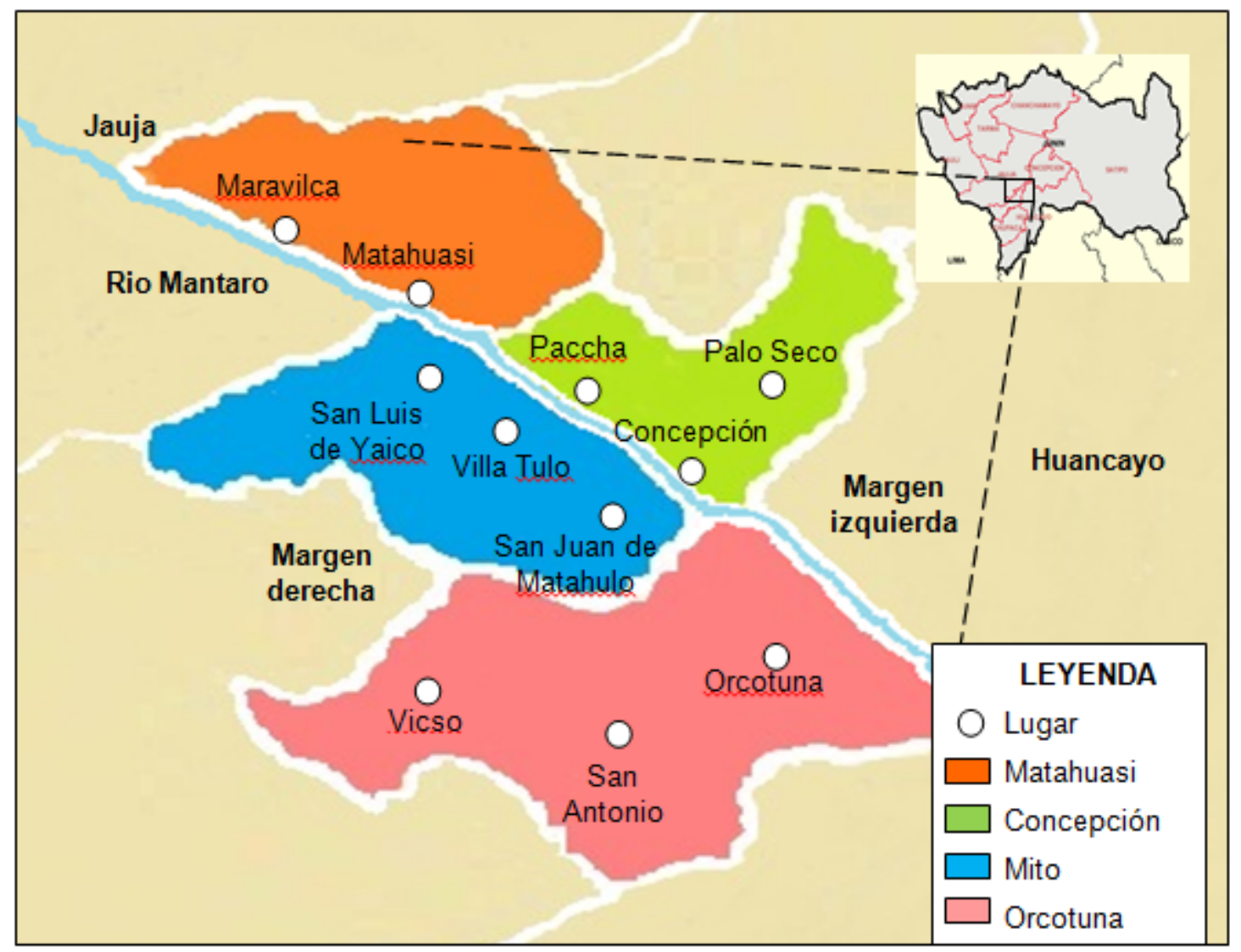

Figura 1. Ubicación de ganaderos lecheros en la provincia de Concepción.

El trabajo se inició reconociendo a los actores del sistema de producción y comercialización de leche fresca a través de dos talleres participativos en la zona; encuestas a 146 ganaderos aplicado entre los meses de agosto a septiembre 2011 (época de $\mathrm{seca}^{4}$ ) en una muestra representativa de poblaciones finitas ( $11 \%$ del total de 1,327 productores); encuestas a 6 acopiadores y 20 procesadores $(34 \%$ del total) y 5 minoristas que permitieron informaciones para estructurar el sistema de producción y comercialización, además se estimaron los costos, considerando el costo de oportunidad de la mano de obra en la zona, y el Margen Bruto de Comercialización (MBC), la Participación del Productor (PDP) y el Margen Neto de Comercialización (MNC) según las siguientes formulas:

$$
\mathrm{MBC}=\left(\frac{\mathrm{Pc}-\mathrm{Pp}}{\mathrm{PC}}\right) * 100
$$

\footnotetext{
${ }^{4}$ Periodo en que casi no existen precipitaciones en la zona, que tienen consecuencia en menor agua para riego y disminución de pastos naturales para alimentación del ganado, por ende, menor producción de leche fresca y mayor precio por el bien.
}

$$
\begin{aligned}
& \qquad \text { PDP }=\left(\frac{P p}{P C}\right) * 100 \\
& \qquad M_{i}=\left(\frac{P_{i}-\left(P_{i-1}+C_{i}\right)}{P C}\right) * 100 \\
& \text { Pc: Precio consumidor. } \\
& \text { Pp: Precio productor. } \\
& \text { Pi: Precio agente i. } \\
& \text { Pi-1: Precio agente i-1 (precio de compra del agente i). } \\
& \text { Ci: Costo de mercadeo. } \\
& \text { Ci: En el productor incluye el costo de producción y }
\end{aligned}
$$

Pc: Precio consumidor.

Pp: Precio productor.

Pi: Precio agente i. Ci: Costo de mercadeo. comercialización y $\mathrm{Po}=0$. 


\section{Resultados y discusión}

Entre los estudios relacionados tenemos el de Espinosa, Rivera y García (2008) sobre la comercialización de leche cruda en México centrado en el desempeño del indicador económico del acopiador, y de Aubron (2006) que resalta las diferencias en el mercadeo entre quesos de leche pasteurizada y de leche cruda en Perú.

En el Valle del Mantaro predomina la pequeña ganadería en la producción de leche fresca. Los productores en general no mantienen contabilidad de costos y sus cálculos obedecen a lógicas distintas de la economía mercantil, donde si bien los valores monetarios asumen relevancia, los valores no monetarios no siempre son incorporados, que dificulta la medición de rentabilidad de la actividad productiva y comercial (Mayer y Glave 1999; Ortiz 2005). En esas economías los costos no siempre se expresan en términos monetarios (Heady 2005).

Huamanchaqui y Porras (2004) encuentran en la zona que $50 \%$ son pequeños ganaderos, el $31 \%$ mediano y el $19 \%$ grande 5 . La proporción de venta de la leche es $96 \%$, y si bien los productores eligen al acopiador, éste último fija el precio. Cortijo, Faure, y Le Gal. (2010) indican que existen diferentes procesadores de lácteos, desde empresas industriales, el Estado (PRONAA) y las queserías artesanales de diversos tamaños. Las empresas industriales pagan un precio más bajo que otros operadores de la zona, pero la seguridad y regularidad en el pago es factor determinante para atraer a los ganaderos, aunque no existan contratos de exclusividad de venta. Las queserías artesanales funcionan de manera informal, sin control sanitario, sin certificación y sin contratos con productores.

Gave (2010) caracteriza en Jauja-Junín el hato ganadero como de tipo mixto (intensivo y extensivo) con tipo de ordeño manual y la alimentación por pastoreo natural. La leche se destina a los porongueros (42\%), la industria Gloria (24\%) y otros destinos (34\%). Bienz (2011) utiliza una herramienta de modelación para optimizar el volumen de producción de leche explorando alternativas en base a los atributos productivos de la finca en tres distritos del Valle de Mantaro.

\subsection{La producción de la leche para la comercialización}

El subsistema de producción comprende: i) El productor; ii) Los eslabonamientos hacia atrás con proveedores de insumos productivos y servicios técnicos; iii) La articulación hacia adelante con la comercialización (Fig. 2). La caracterización del productor ganadero permite clasificarlo en tres grupos ${ }^{6}$ : pequeño $(59 \%)$ que poseen 2 vacas en ordeño de un rebaño de 6 cabezas, producen 16.9 $\mathrm{L} /$ leche por fundo $\mathrm{y}$ el rendimiento promedio es $9.7 \mathrm{~L} /$ vaca, mediano (31\%) y grande (10\%). En los pequeños ganaderos el tipo de raza predominante es

\footnotetext{
${ }_{5}^{5}$ El pequeño productor tiene menos de 4 vacunos con 2 vacas en ordeño, el mediano posee de 5 a 9 vacunos con 2 vacas en ordeño y el grande más de 10 vacunos con 7 vacas en ordeño.

${ }^{6} \mathrm{Se}$ clasificó con el análisis cluster en base a cuatro características: Número de vacunos, número de vacas en ordeño, producción del fundo a día y rendimiento por vaca al día.
}

Criollo y Holstein, el ordeño es manual y una vez al día. En las medianas explotaciones predominan razas Holstein, Brown Swiss y el ordeño manual. En grandes explotaciones predominan las razas Holstein y Brown Swiss, el ordeño es manual y mecánico. La mayoría de productores utilizan la inseminación artificial (Tabla 2), pero sólo el $16 \%$ de productores se encuentran organizados en asociaciones ${ }^{7}$.

Las articulaciones hacia atrás comprenden a los proveedores de insumos productivos (semillas para pastos, abonos, minerales y concentrados) y los abastecedores de insumos técnicos (capacitación, asistencia técnica veterinaria y financiamiento) (Fig. 2).

Los forrajes son producidos en la finca del productor y en pocos casos son preservados como ensilaje o heno. El tipo de forraje utilizado depende de las condiciones de suelo y clima ${ }^{8}, 97 \%$ de productores adquieren semillas para pastos en las tiendas de Huancayo que en algunos casos se desplazan a los centros de producción. En abonos se emplean urea, fosfato, cloruro de sodio y nitrato comprados por más del $94 \%$ de productores en Concepción y Huancayo. La sal (mineral, negra y común) es requerida en cantidades pequeñas, se incluyen como premezclado en el concentrado, son adquiridas por $88 \%$ de productores en Concepción y Huancayo. $62 \%$ de los productores acceden a los concentrados que permiten formular dietas para maximizar la producción lechera.

Sólo 29\% de productores acceden a la capacitación, $33 \%$ de ellos pequeños, $29 \%$ medianos y $13 \%$ grandes $^{9}$, las instituciones que la realizan son públicas y privadas en temas de manejo de pastos, mejoramiento genético, sanidad, alimentación del ganado, ordeño. El 94\% de productores acceden a servicios veterinarios que provienen $97 \%$ de privados y $3 \%$ del sector público que evidencia el abandono del Estado a la asistencia en la zona. El 19\% de productores acceden a préstamos bancarios para inversión en la actividad lechera, en entidades privadas como la Financiera Confianza (54\%), la Caja Huancayo (14\%), Mi Banco (7\%), otros (25\%). El $79 \%$ de los productores que toman préstamos es por monto menor a S/. 5,000 Soles, el 14\% obtiene préstamos mayores a S/. 10,000 y el 7\% oscilan en el rango de S/. 5,000 hasta $\mathrm{S} / .10,000$

\subsection{Sistema de comercialización de leche fresca y derivados lácteos}

Los principales intermediarios de la comercialización son:

Acopiador rural: Recolectan diariamente la leche fresca para abastecer a los procesadores, habiéndose ${ }^{724 \%}$ de la margen derecha (Asociación: San Luis de Yaico, San Juan de Matahulo y Villatulo) y $26 \%$ en la margen izquierda (Asociación: Asunción, Maravilca, Palo Seco y Señor de los Milagros).

${ }^{8}$ Los pastos cultivados son avena vicia, asociación simple (Trébol rojo y Rye grass italiano) y pastoreo (Rye grass ingles + Rye gras italiano + Dáctilis glomerata + Trébol rojo + Trébol blanco).

${ }^{9}$ En los grandes productores la asesoría la realiza algún miembro de la familia, siendo técnico agropecuario o Ing. Zootecnista, pero sí buscan capacitación en mejoramiento genético. 
identificado tres tipos de ellos: i) Aquel que sólo acopia de los productores; ii) El que acopia de los productores y de otro acopiador para la reventa; iii) Es acopiador y también procesador. El 83\% de acopiadores son locales y $17 \%$ proviene de Huancayo. El acopiador de Matahuasi recolecta $66 \%$ de leche fresca $(2,900 \mathrm{~L} /$ día $)$, el de Sicaya $23 \%$ (1,000 L) y el de Orcotuna 11\% (480 L). Los envases empleados son bidones de plástico con tapa y capacidad de $100 \mathrm{~L}$ que se trasladan $65 \%$ en camionetas, $15 \%$ en moto, $10 \%$ en bicicleta y $10 \%$ a pie. Los precios pagados y recibidos se muestran en la tabla 3 .
Las articulaciones hacia adelante son con la comercialización donde el pequeño productor canaliza $87 \%$ de leche fresca a la venta y $13 \%$ al procesamiento propio y consumo familiar. El mediano productor destina $91 \%$ a la venta y $9 \%$ al consumo propio, en el grande productor el $97 \%$ se destina a la venta y $3 \%$ al autoconsumo (Fig. 2). Esas relaciones varían entre la margen derecha y la margen izquierda del río Mantaro, estos últimos poseen mayor rendimiento y están más vinculados al sistema comercial.

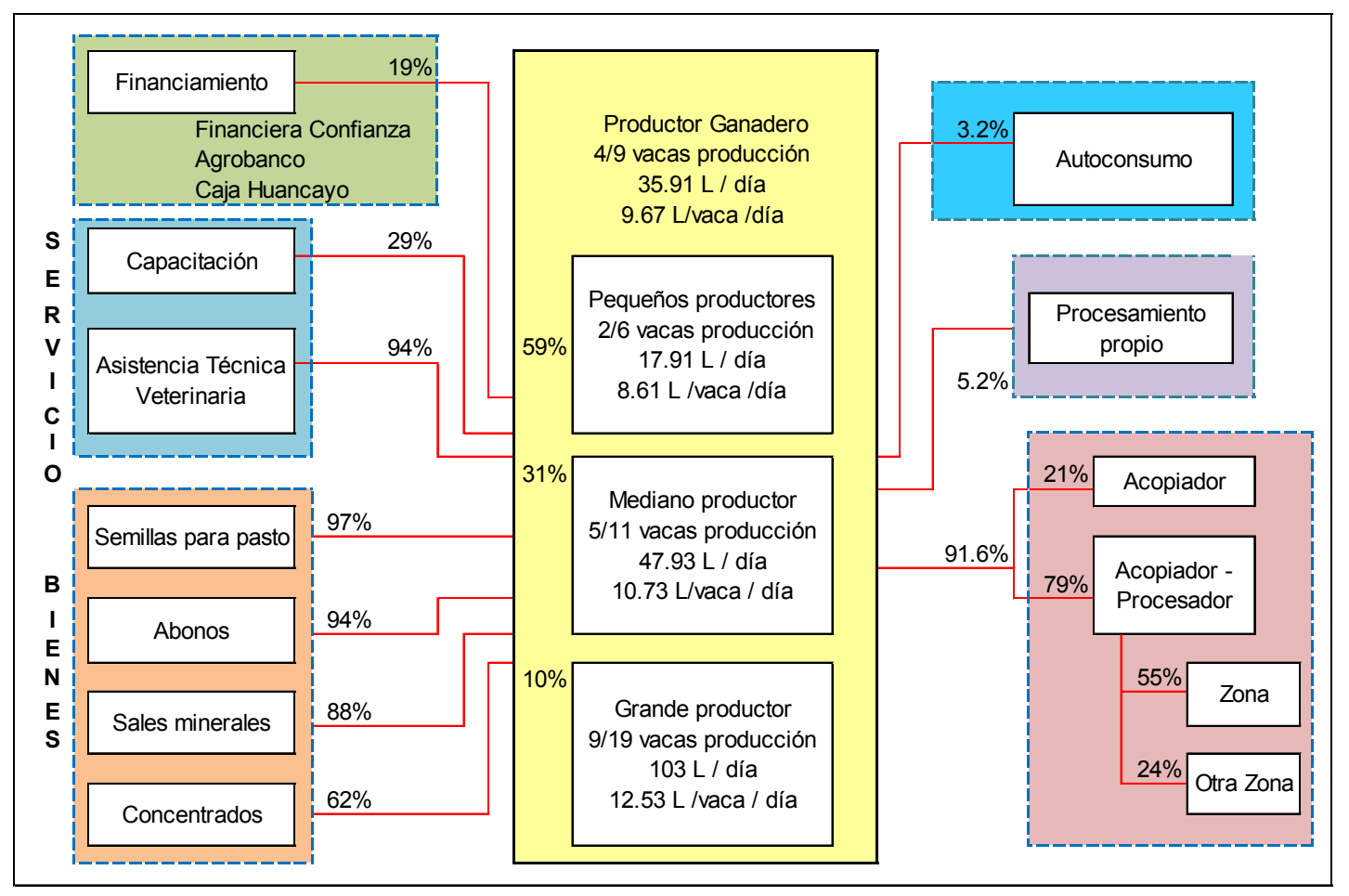

Figura 2. El subsistema de producción de la leche para el periodo de seca.

Tabla 2. Tecnología del productor ganadero (época de seca).

\begin{tabular}{|c|c|c|c|c|c|c|c|c|}
\hline \multirow{2}{*}{$\begin{array}{l}\text { Nivel tecnológico del } \\
\text { productor }\end{array}$} & \multicolumn{2}{|c|}{$\begin{array}{l}\text { Pequeño } \\
\text { Productor }\end{array}$} & \multicolumn{2}{|c|}{$\begin{array}{l}\text { Mediano } \\
\text { Productor }\end{array}$} & \multicolumn{2}{|c|}{$\begin{array}{l}\text { Grande } \\
\text { Productor }\end{array}$} & \multicolumn{2}{|c|}{ Total } \\
\hline & $\mathrm{N}^{\circ}$ & $\%$ & $\mathrm{~N}$ & $\%$ & $\mathrm{~N}$ & $\%$ & $\mathrm{~N}$ & $\%$ \\
\hline \multicolumn{9}{|l|}{ Número de Ordeño } \\
\hline Una vez al día & 56 & $65 \%$ & 10 & $22 \%$ & 1 & $7 \%$ & 67 & $46 \%$ \\
\hline $\begin{array}{l}\text { Dos veces al día } \\
\text { Tipo de Ganado }\end{array}$ & 30 & $35 \%$ & 35 & $78 \%$ & 14 & $93 \%$ & 79 & $54 \%$ \\
\hline Criollo & 43 & $37 \%$ & 19 & $26 \%$ & 1 & $5 \%$ & 63 & $29 \%$ \\
\hline Brown Swiss & 31 & $27 \%$ & 23 & $31 \%$ & 8 & $36 \%$ & 62 & $28 \%$ \\
\hline Holstein & 41 & $36 \%$ & 32 & $43 \%$ & 13 & $59 \%$ & 86 & $39 \%$ \\
\hline Estabulado & 7 & $8 \%$ & 10 & $22 \%$ & 3 & $20 \%$ & 20 & $14 \%$ \\
\hline $\begin{array}{l}\text { Tipo de Ordeño } \\
\text { Manual }\end{array}$ & 86 & $100 \%$ & 45 & $100 \%$ & 14 & $93 \%$ & 145 & $99 \%$ \\
\hline Mecánico & 0 & $0 \%$ & 0 & $0 \%$ & 1 & $7 \%$ & 1 & $1 \%$ \\
\hline Inseminación artificial & 50 & $58 \%$ & 40 & $89 \%$ & 14 & $93 \%$ & 104 & $71 \%$ \\
\hline
\end{tabular}


Procesador: Se encontró diversidad de transformadores como la gran industria Gloria, el programa social PRONAA $^{10}$ y el procesador artesanal de queso fresco ${ }^{11}$. Los procesadores formales son especializados y tienen acopiadores propios, mantienen relaciones de confianza con el productor caracterizado por el pago puntual, además compran a otros acopiadores. Los transformadores artesanales realizan más de una actividad $(80 \%$ es acopiador-transformador, $15 \%$ productor-acopiadortransformador y $5 \%$ productor-transformador). Se paga a los productores entre S/. 1.0 hasta S/. 1.1 por L, y a los acopiadores desde S/. 1.17 hasta S/. 1.35 por L. Los informales ofrecen mayor precio pero no realizan análisis continuo de la calidad de la leche fresca entregada por el ganadero. La materia prima se convierte en leche pasteurizada y diversos derivados lácteos (Tabla 4).

El distrito de Concepción evidencia diversificación en derivados lácteos debido a la mayor inversión en maquinarias y acceso a la capacitación, en los otros distritos que se especializan principalmente en la producción de queso fresco y yogurt su inversión en equipos es reducida y existe poco conocimiento en elaboración de otros derivados. El queso fresco producido por día fue de $1,968 \mathrm{~kg}$ y su precio oscila entre S/. 9.8 a $\mathrm{S} / .13 .0$ y la tasa de conversión entre 6.8 y $7.5 \mathrm{~L} /$ leche por $\mathrm{kg}$ de queso. El volumen de yogurt es de 1,631 L a precio promedio entre S/. 3.0 y S/. 3.7 L.

Mercadeo minorista: El destino del queso fresco fue $12 \%$ hacia Huancayo (mercado Modelo y supermercado Plaza Vea), los mercados regionales (Jauja y La Oroya, Pasco), y las ferias locales; el 88\% hacia Lima, de ellos $71 \%$ al mercado minorista y $17 \%$ a los supermercados ${ }^{12}$ (Ewong y Metro) (Tabla 5).

En el mercado minorista de Lima existe gran cantidad de comercializadores (25 puestos) de queso fresco que provienen de Huancayo, Huarochirí, Huancavelica, Yauyos y Chincha, muy pocos tienen marcas, y más bien utilizan etiquetas como quesos provenientes de Huarochirí1 ${ }^{13}$. La provisión es por encomienda o compra directa en la zona productora, y el precio de venta $\mathrm{S} /$. $10 / \mathrm{kg}$, desde allí se dirige a otros mercados minoristas ${ }^{14}$ $(90 \%)$ y a consumidores finales $(10 \%)$.

El volumen total comercializado de leche fresca es $22,604 \mathrm{~L} /$ día, el $30 \%$ es leche procesada, 3\% leche fresca, $61 \%$ queso fresco y $9 \%$ otros derivados (manjar,

${ }^{10}$ Programa del Estado peruano para alimentación en colegios de la zona, es abastecido por procesadores de $3010 \mathrm{~L} /$ leche/día (precio pactado mediante contrato).

${ }^{11}$ De las 20 plantas procesadoras, 15 son artesanales y 5 son formales (Sierra Verde, El Valle, El Mantaro, CONCELAC) y Matahuasi (El Cesar's).

${ }^{12}$ Los supermercados exigen quesos de mejor calidad, elaborados con leche pasteurizada y prensados, con marcas y etiquetas que son proveídos por las industrias formales de la zona. Los quesos artesanales frecuentemente son transformados directamente de la leche cruda.

${ }^{13}$ Los quesos de Matahuasi cambian su nombre de origen durante la comercialización, es queso Junín si se vende en los mercados regionales, en Lima es llamado "queso Huarochirano".

${ }^{14}$ Entre ellos, Jorge Chávez, mercado de Productores, mercado Central, mercado Ciudad de Dios. mantequilla, yogurt y helados). La Figura 3 y Tabla 6 muestran los canales de comercialización de la leche (fresca y procesada) y queso fresco que representan el $83 \%$ del total producido.

Canales leche procesada: Los dos primeros canales van hacia la industria procesadora a través sus propios acopiadores y explican $27 \%$ del flujo total. El primero es cuando los recolectores de Gloria trasladan la leche a su centro de acopio en Concepción, donde se la enfría antes de llevarse a la planta procesadora en Huachipa (Lima), el producto es leche evaporada. El segundo es el acopio por concesionarios del programa social PRONAA ${ }^{15}$ que recolectan la leche con sus propios acopiadores y otros, y procesan

Tabla 3. Precio de compra y venta de la leche fresca (S/. / L).

\begin{tabular}{lcccc}
\hline \multirow{2}{*}{ Distrito } & \multicolumn{2}{c}{$\begin{array}{c}\text { Precio de } \\
\text { compra }\end{array}$} & \multicolumn{3}{c}{ Precio de venta } \\
\cline { 2 - 5 } & al productor & PRONAA & Gloria SA. Mercado \\
\hline Orcotuna & 1.01 & & & 1.47 \\
Matahuasi & 1.09 & 1.20 & 1.06 & \\
Sicaya & 1.05 & 1.15 & & \\
Promedio & 1.05 & 1.18 & 1.06 & 1.47 \\
\hline \multicolumn{2}{l}{ Fuente: Encuesta a acopiadores, setiembre 2011 }
\end{tabular}

Canales de queso fresco: Los canales 3 al 11 explican $53.3 \%$ del flujo total de leche fresca que van a través acopiadores a los procesadores artesanales (48.6\%) $\mathrm{y}$ otros $(4.7 \%)$, son transformados en queso fresco y enviados a Lima (37.6\% al mercado Minorista y el $8.4 \%$ a supermercados Ewong y Metro), a Huancayo (4.6\% al mercado Modelo y $1.6 \%$ al supermercado Plaza Vea) y $1.1 \%$ a la feria local (Aco y Huancayo) y regional (Huánuco).

Canales leche fresca: El Canal 12 es la comercialización de leche fresca que se obtiene de los acopiadores y se vende en el Mercado Modelo de Huancayo. El precio pagado es $\mathrm{S} /$. 1.47 L, el producto es altamente perecedero y no sometido a ningún proceso previo.

Los canales 1 al 11 corresponden al mercadeo centralizado impulsado por las plantas procesadoras (gran industria y empresas formales e informales) que se relacionan con los productores a través el acopio de leche fresca (72.8\% del volumen acopiado) y con los detallistas (mercados mayoristas, minoristas y ferias) en la distribución de leche procesada y queso fresco $(80.5 \%$ del volumen producido). El canal 12 corresponde al comercio directo de leche fresca (acopiador-consumidor) o mercadeo descentralizado $(2.8 \%$ del total $)$.

\footnotetext{
${ }^{15}$ En el periodo de estudio seis empresas ganaron la concesión del programa social PRONAA para elaborar leche pasteurizada dirigida a los colegios de la región Junín.
} 
Tabla 4. Producción de derivados lácteos del procesador artesanal.

\begin{tabular}{|c|c|c|c|c|c|c|c|c|c|c|c|c|c|c|}
\hline \multirow{3}{*}{$\begin{array}{l}\text { Procesador del } \\
\text { distrito }\end{array}$} & \multicolumn{14}{|c|}{ Comercialización de derivados lácteos por día } \\
\hline & \multicolumn{2}{|c|}{ Leche } & \multicolumn{2}{|c|}{ Queso } & \multicolumn{2}{|c|}{ Yogurt } & \multicolumn{2}{|c|}{ Mantequilla } & \multicolumn{2}{|c|}{ Manjar } & \multicolumn{2}{|c|}{ Helados } & \multicolumn{2}{|c|}{ Total } \\
\hline & $\mathrm{N}$ & $\%$ & $\mathrm{~N}$ & $\%$ & $\mathrm{~N}$ & $\%$ & $\mathrm{~N}$ & $\%$ & $\mathrm{~N}$ & $\%$ & $\mathrm{~N}$ & $\%$ & $\mathrm{~N}$ & $\%$ \\
\hline$\overline{\text { Orcot }}$ & & & 2 & $50 \%$ & 2 & $50 \%$ & & & & & & & 4 & $100 \%$ \\
\hline Mito & & & 1 & $100 \%$ & & & & & & & & & 1 & $100 \%$ \\
\hline Concep & 2 & $8 \%$ & 6 & $24 \%$ & 7 & $28 \%$ & 4 & $16 \%$ & 4 & $16 \%$ & 2 & $8 \%$ & 25 & $100 \%$ \\
\hline Matahuasi & & & 9 & $64 \%$ & 5 & $36 \%$ & & & & & & & 14 & $100 \%$ \\
\hline Total & 2 & $5 \%$ & 18 & $41 \%$ & 14 & $32 \%$ & 4 & $9 \%$ & 4 & $9 \%$ & 2 & $5 \%$ & 44 & $100 \%$ \\
\hline
\end{tabular}

Fuente: Encuesta a acopiadores - procesadores, setiembre 2011

Tabla 5. Comercialización de queso en Lima (por día).

\begin{tabular}{lcccccc}
\hline \multirow{2}{*}{$\begin{array}{c}\text { Distrito de } \\
\text { Procedencia }\end{array}$} & \multicolumn{2}{c}{ Mercado Minorista } & \multicolumn{2}{c}{ Supermercados } & \multicolumn{2}{c}{ Total } \\
\cline { 2 - 7 } & $\mathrm{Kg}$ & $\%$ & $\mathrm{Kg}$ & $\%$ & $\mathrm{~N}$ & $\%$ \\
\hline Orcotuna & 55 & $4 \%$ & & & 55 & $3 \%$ \\
Concepción & 232 & $17 \%$ & & & 232 & $13 \%$ \\
Matahuasi & 1,110 & $79 \%$ & 340 & $100 \%$ & 1,450 & $83 \%$ \\
\hline \multicolumn{1}{c}{ Total } & 1,397 & $100 \%$ & 340 & $100 \%$ & 1,737 & $100 \%$ \\
\hline
\end{tabular}

Fuente: Encuesta a acopiadores - procesadores, setiembre 2011

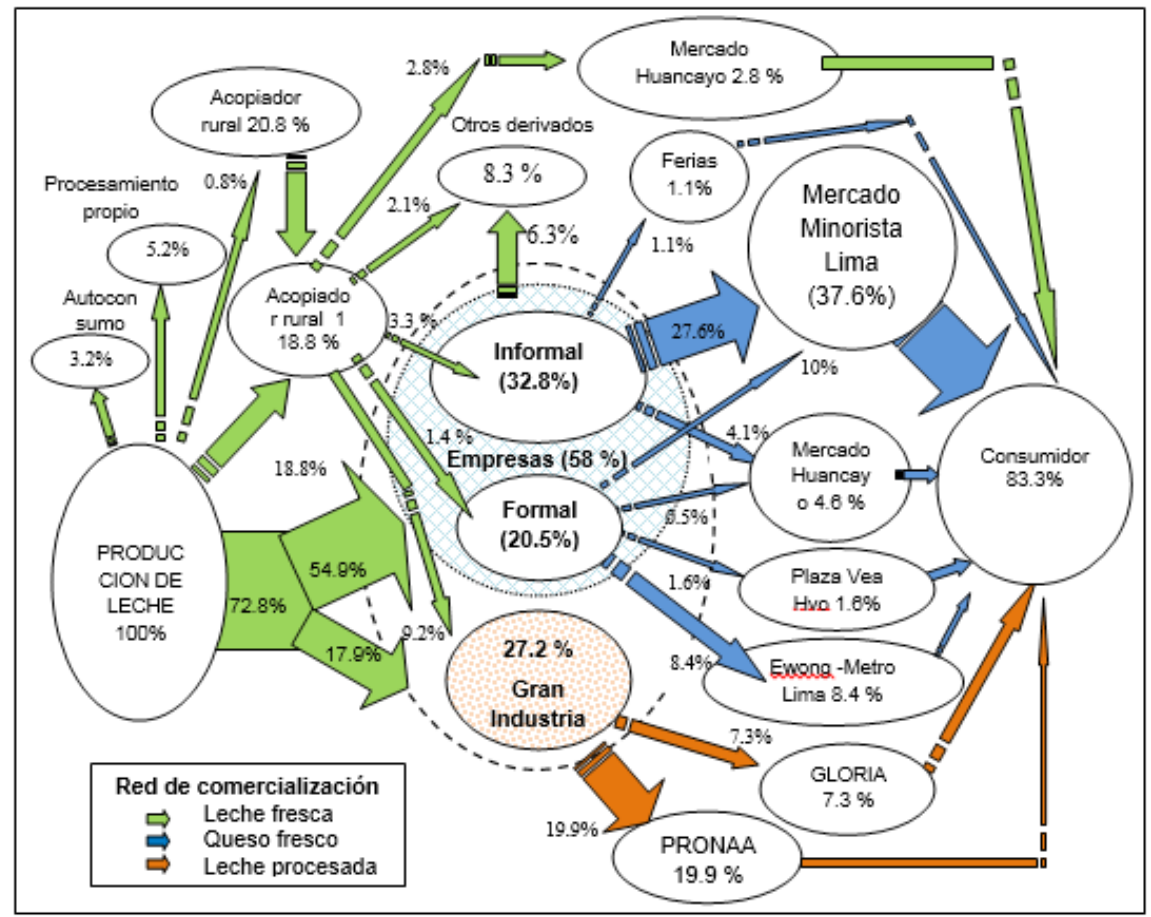

Figura 3. Red de comercialización de leche y queso fresco (periodo de seca, 2011). 
Tabla 6. Red de comercialización de la leche y queso fresco al día (periodo de seca, 2011).

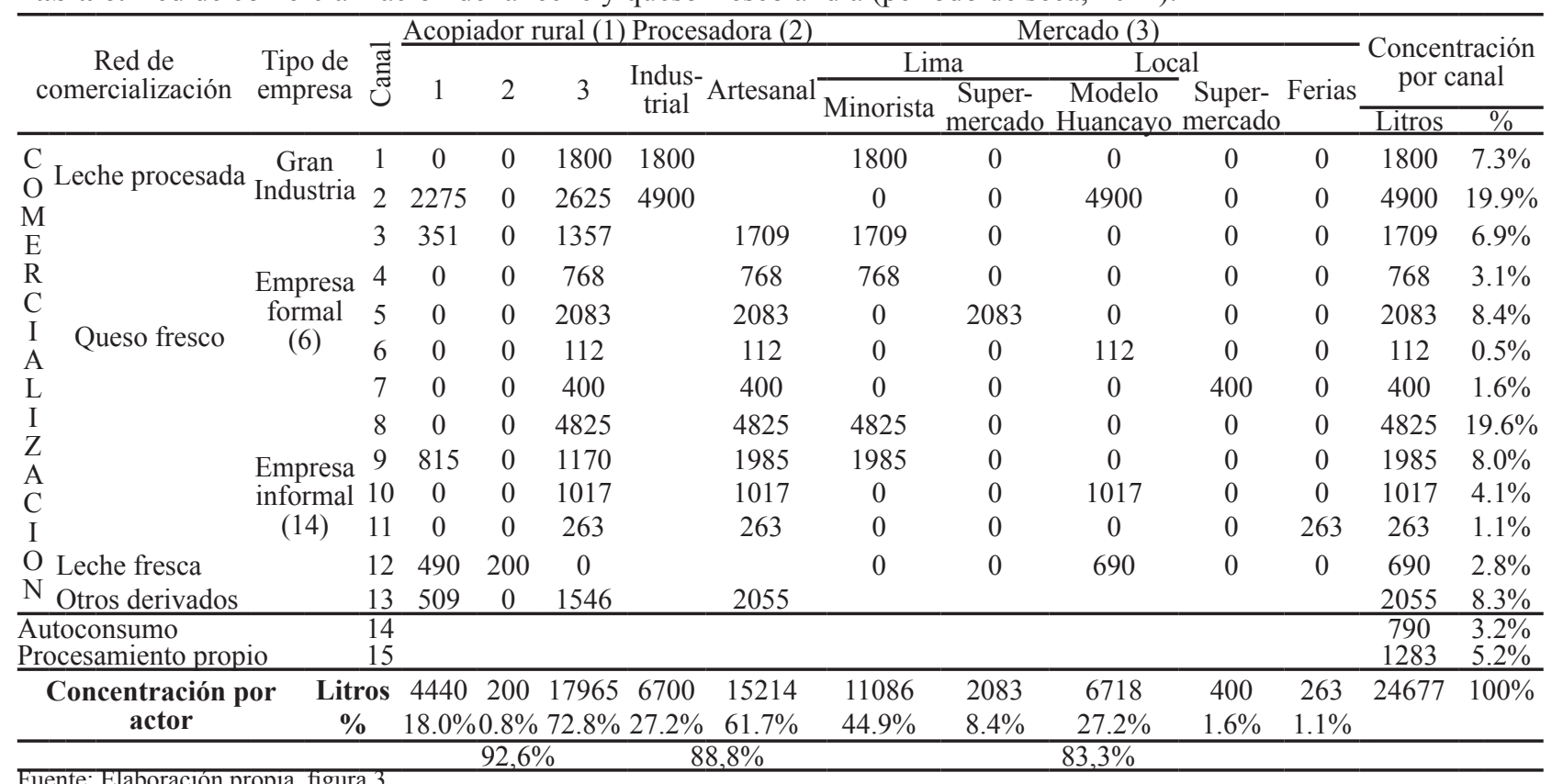

Nota: El canal 13 es la comercialización de leche en otros derivados lácteos y los canales 14 y 15 es la destinada al autoconsumo y procesamiento.

(1) Acopiador rural: El primero sólo compra de leche del productor ganadero, el segundo vende al acopiador 1 y el tercero es un acopiador-transformador.

(2) Procesadores: Gran industria (empresa Gloria S. A. y programa social PRONAA), seis empresas pequeñas formales y catorce empresas artesanales.

(3) Mercado: Supermercado local (Plaza Vea de Huancayo) y en Lima (Ewong y Metro), feria local (Aco y Huancayo) y regional (Huánuco).

1.3 Costos, precios y márgenes de comercialización a) Costos de producción: En la zona de estudio las explotaciones ganaderas son muy heterogéneas lo que genera diferencias en los costos de producción ${ }^{16}$. Para un pequeño productor con 4 vacas en producción con 38.5 L/leche/día y total de 6 animales, el costo por L/leche fresca es 0.95 Soles, desagregado en alimentación $57 \%$, servicio veterinario $5 \%$, materiales y maquinarias $1 \%$, mano de obra ${ }^{17} 29 \%$, gastos financieros y depreciación $8 \%$. El grande productor con 14 vacas en producción con $252 \mathrm{~L} /$ leche/día y un total de 33 animales, el costo por L/ leche es 0.94 Soles, desagregados en alimentación 79\%, servicios veterinarios $2 \%$, materiales y maquinarias ${ }^{18}$ $3 \%$, mano de obra $16 \%$ (dos personas), depreciación $1 \%$ (Tabla 7).

b) Costos de acopio: Tienen tres componentes: Materiales (3.2\% en bidones de plástico), transporte $93.0 \%$ y mano de obra $(3.8 \%)$. El costo de acopio por L/leche fresca es 0.03 soles $^{19}$ (Tabla 7 ).

c) Costos de procesamiento de queso fresco: Las plantas procesadoras de queso fresco difieren en volumen de leche acopiado, herramientas y tenencia de registros sanitarios que diferencian las empresas formales e ${ }^{16}$ Obtenidos por entrevistas a profundidad a ganaderos representativos de tipo pequeño (en distrito El Mantaro) y grande (en el distrito de Matahuasi).

${ }^{17}$ Valuado en $90 \%$ del precio del mercado (jornal) como costo de oportunidad debido al dinamismo del mercado de trabajo local en ese sistema productivo.

${ }^{18} \mathrm{El}$ grande productor utiliza manteles, carretilla, lampa, cal, sellador (ordeño), máquina para tetillas y ordeñadora, alimenta el ganado con concentrados y tiene personal contratado.

${ }^{19}$ Registro de acopiadores (septiembre 2011).

232 informales. Estos costos se agrupan en insumos (cuajo, sal, cloruro de calcio, cloruro, flete) que son $17.5 \%$, energía (gas o leña) $28.5 \%$, equipos y maquinarias (tinas, moldes de acero inoxidable o paja, marmitas, agitador, colador, mesas, manteles, javas, balanza electrónica) $10.2 \%$, materiales de limpieza $1.2 \%$, intereses $0.2 \%$ y mano de obra $42.3 \%$ (Tabla 7 ).

d) Costos de mercadeo: Incluyen servicios (alquiler del local, energía, agua, luz y telefonía), materiales de limpieza, equipos y herramientas (congelador, balanzas, etc.), mano de obra, transporte, carnet de sanidad e infraestructura (Tabla 7). En el comercio de queso fresco los costos en el mercado Modelo de Huancayo es S/. 0.57 $\mathrm{kg}$ y en el mercado Minorista de Lima S/. $0.30 \mathrm{~kg}$ debido a diferencias en el alquiler del puesto de venta (300 Soles/ mes en Huancayo y 17 Soles/mes en Lima) ${ }^{20}$. En ambos mercados los detallistas se dedican más de ocho horas al comercio y sólo los comerciantes del mercado de Lima presentan Carnet de Sanidad. En la leche fresca el costo unitario es $\mathrm{S} /$. $0.15 \mathrm{~L}$, el mayor gasto es transporte pues el minorista acopia la leche y lo lleva hasta el centro de venta $^{21}$.

e) Precios en los agentes: Los precios usados para estimar los márgenes de comercialización han sido recopilados en el campo para cada actor de la cadena de comercialización durante el periodo de seca (Tabla 7).

${ }^{20}$ Entrevistas a comerciantes minoristas en el mercado Modelo de Huancayo y el mercado Minorista de Lima (septiembre 2011).

${ }^{21} \mathrm{La}$ comercialización de leche fresca en el mercado Modelo de Huancayo se realiza afuera y/o en los alrededores del mercado, se paga S/. 2,00 por día para limpieza del área empleada. 
f) Margen Bruto de Comercialización (MBC): $\mathrm{Se}$ estima el $\mathrm{MBC}$ en siete canales que representan $49 \%$ del volumen total ${ }^{22}$ (Tabla 8 ). En el canal 3, por cada S/. 100 pagado por el consumidor, $60.1 \%$ le corresponden al ganadero (PDP) y 39.9\% a la intermediación, distribuida en $9.0 \%$ al acopiador, $23,3 \%$ al procesador formal y $7.6 \%$ al minorista de Lima. En el canal 4, a la planta procesadora formal le corresponde el $32.4 \%$ al realizar también el acopio. En el canal 6 donde la producción es comercializada en Huancayo, la PDP y el MBC son similares al obtenido por el canal 3, pues la venta se realiza en la propia tienda de cada procesadora formal.

En el canal 8, por cada S/. 100 pagado por el consumidor, $69.2 \%$ le corresponden al productor (PDP) y $30.8 \%$ a la intermediación, distribuida en $18.7 \%$ al procesador informal y $12.1 \%$ al minorista de Lima. En el canal 9, a la planta procesadora informal le corresponde $8,3 \%$ pues ella no acopia la leche (poco frecuente en la zona de estudio). Para el canal 10, la PDP es menor cuando vende directamente a las plantas procesadoras informales.

En la comercialización de la leche fresca 12A, la PDP es mayor en relación a otros canales, pues el $75.3 \%$ le corresponde al productor y $24.7 \%$ a la intermediación. En el canal 12B el minorista que acopia y comercializa recibe además del MBC minorista, el MBC del acopiador.

g) Margen Neto de Comercialización (MNC): Es obtenido por cada agente (Tabla 9). Para el canal 3 el total de utilidad neta del mercadeo fue $28.3 \%$. Los productores pequeños obtienen $9.5 \%$ de beneficio y los grandes el $5.6 \%$ y el procesador formal $15.7 \%$ (mayor de todos).

En el canal 4 se incrementa el MNC del procesador formal a $24.7 \%$ al acopiar ellos mismos la leche fresca, pero cuando el queso fresco pasteurizado se comercializa en Huancayo (canal 6), los MNC son $26.4 \%$ y $31.2 \%$ según se incluye al pequeño o grande productor. El canal 4 presenta un menor costo de mercadeo total pues hay un menor número de agentes y por ende un mayor margen neto en la cadena de $30.2 \%$.

El canal 8 ocurre si el queso fresco lo vende un procesador informal, el $\mathrm{MN}$ del productor es $11 \%$ para el pequeño y $6.4 \%$ para el grande productor y del procesador $10.6 \% \mathrm{y}$ $16.1 \%$ respectivamente. En el canal 10, el MNC es $24.4 \%$ y $29.4 \%$ según participa el pequeño o grande productor, debido a la ausencia de acopiadores y a los menores costos del procesador informal.

En el mercadeo de leche fresca en el mercado de Huancayo, el pequeño productor obtiene mayor beneficio neto de $11.9 \%$, frente al acopiador y minorista que tienen $9.1 \%$ y $3.3 \%$ de beneficio (canal 12A), pero el grande productor obtiene menores ganancias unitarias que el pequeño. El acopiador - minorista del canal 12B incrementa su beneficio unitario a $20.7 \%$ si asume la recolección de leche fresca. Estos canales directos son

\footnotetext{
${ }^{22}$ Los canales $1,2,5$ y 7 no se incluyen debido a la confidencialidad de la información de Gloria S. A. y los Supermercados Ewong y Metro en Lima y Plaza Vea de Huancayo.
}

muy poco representativos del comercio total $(2.8 \%)$.

h) La rentabilidad: En el comercio de leche y queso fresco, la rentabilidad con escala de operación determina el beneficio neto de cada agente como proporción de los costos (en Soles). El pequeño productor obtiene en todos los canales una menor ganancia neta de S/. 84 inferior al grande productor de S/. 313 mensual.

El acopiador obtiene mayor ganancia al recolectar leche de grandes productores y venderlos al procesador. La ganancia del transformador es mayor para la empresa formal (entre 13,412 y 8,508 Soles/mes) y menor para el informal $(2,695$ a 49 Soles/mes). En la leche fresca el minorista obtiene mayores ganancias si el mismo recolecta el producto. La planta (formal e informal) obtiene mayores ganancias cuando asume la función de acopio (Tabla 10 y 11).

Tabla 7. Costos, precios promedio de leche fresca y queso fresco.

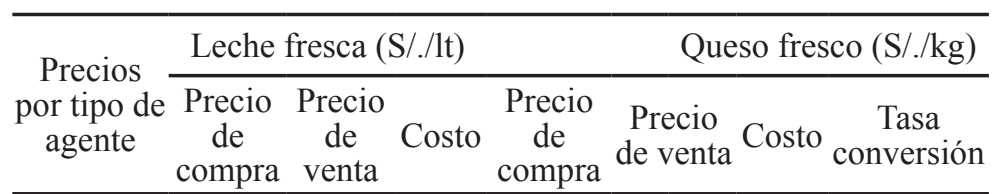

\begin{tabular}{ll}
\hline $\begin{array}{l}\text { Pequeño } \\
\text { productor }\end{array}$ & $\mathrm{S} / .1 .130 .95$ \\
$\begin{array}{l}\text { Grande } \\
\text { productor }\end{array}$ & $\mathrm{S} / .1 .040 .94$
\end{tabular}

Acopiador $\mathrm{S} / .1 .06 \mathrm{~S} / .1 .300 .03$

rural

S/. $1.06 \mathrm{~S} / .1 .300 .03$

Procesador $\mathrm{S} / .1 .20$

Procesador

informal $\mathrm{S} / .1 .06$

S/. $9.67 \quad 0.89$

6.74

Mercado

Minorista

$\mathrm{S} / .9 .50 \mathrm{~S} / .11 .00 \quad 0.30$

Lima

Mercado

Modelo

Huancayo

Detallista

Huancayo

y Lima

Mercado

Modelo S/. 1.20 S/. 1.500 .15

Huancayo

Fuente: Elaboración propia, entrevistas a productores y agentes comerciales 2011 
Tabla 8. PDP y MBC del queso fresco y la leche fresca.

\begin{tabular}{ccccccc}
\hline \multirow{2}{*}{ Canal } & $\begin{array}{c}\text { Participación del } \\
\text { productor (\%) }\end{array}$ & \multicolumn{3}{c}{ Margen Bruto de Intermediación (\%) } & \multirow{2}{*}{ Acopiador } \\
\hline \multirow{2}{*}{3} & PP & 60.1 & 9.0 & 23.3 & 7.6 & 39.9 \\
& GP & 55.3 & 13.8 & 23.3 & 7.6 & 44.7 \\
4 & PP & 60.1 & & 32.4 & 7.6 & 39.9 \\
& GP & 55.3 & & 37.2 & 7.6 & 44.7 \\
6 & PP & 60.1 & 9.0 & 23.3 & 7.6 & 39.9 \\
& GP & 55.3 & 13.8 & 23.3 & 7.6 & 44.7 \\
8 & PP & 69.2 & & 18.7 & 12.1 & 30.8 \\
& GP & 63.7 & & 24.2 & 12.1 & 36.3 \\
9 & PP & 69.2 & 10.4 & 8.3 & 12.1 & 30.8 \\
& GP & 63.7 & 15.9 & 8.3 & 12.1 & 36.3 \\
10 & PP & 63.5 & & 17.1 & 19.4 & 36.5 \\
& GP & 58.4 & & 22.2 & 19.4 & 41.6 \\
$12 \mathrm{~A}$ & PP & 75.3 & 11.3 & & 13.3 & 24.7 \\
& GP & 69.3 & 17.3 & & 13.3 & 30.7 \\
$12 B$ & PP & 75.3 & & & 24.7 & 24.7 \\
& GP & 69.3 & & & 30.7 & 30.7 \\
\hline PP. Pequeño
\end{tabular}

PP: Pequeño productor y GP: Grande productor.

Fuente: Elaboración propia con referencias de los tablas 6, 7 y figura 3.

Tabla 9. MNC del queso fresco y la leche fresca.

\begin{tabular}{|c|c|c|c|c|c|c|}
\hline \multirow{2}{*}{\multicolumn{2}{|c|}{ Canal }} & \multirow{3}{*}{$\begin{array}{l}\text { Margen Neto al } \\
\text { productor }(\%)\end{array}$} & \multicolumn{3}{|c|}{ Margen Neto a la Intermediación (\%) } & \multirow{2}{*}{ Total } \\
\hline & & & Acopiador & Procesador & Minorista & \\
\hline \multirow[b]{2}{*}{3} & $\mathrm{PP}$ & & 7.2 & 15.7 & 5.4 & 28.3 \\
\hline & GP & 5.6 & 12.0 & 15.7 & 5.4 & 33.1 \\
\hline \multirow{2}{*}{4} & PP & 9.5 & & 24.7 & 5.4 & 30.2 \\
\hline & GP & 5.6 & & 29.5 & 5.4 & 34.9 \\
\hline \multirow{2}{*}{6} & PP & 9.5 & 7.2 & 15.7 & 3.5 & 26.4 \\
\hline & GP & 5.6 & 12.0 & 15.7 & 3.5 & 31.2 \\
\hline \multirow{2}{*}{8} & PP & 11.0 & & 10.6 & 9.4 & 20.0 \\
\hline & GP & 6.4 & & 16.1 & 9.4 & 25.5 \\
\hline \multirow{2}{*}{9} & PP & 11.0 & 8.3 & 0.2 & 9.4 & 17.9 \\
\hline & GP & 6.4 & 13.8 & 0.2 & 9.4 & 23.4 \\
\hline \multirow{2}{*}{10} & $\mathrm{PP}$ & 10.1 & & 9.7 & 14.7 & 24.4 \\
\hline & GP & 5.9 & & 14.8 & 14.7 & 29.4 \\
\hline \multirow{2}{*}{$12 \mathrm{~A}$} & PP & 11.9 & 9.1 & & 3.3 & 12.4 \\
\hline & GP & 7.0 & 15.1 & & 3.3 & 18.4 \\
\hline \multirow{2}{*}{$12 \mathrm{~B}$} & PP & 11.9 & & & 14.7 & 14.7 \\
\hline & GP & 7.0 & & & 20.7 & 20.7 \\
\hline
\end{tabular}

PP: Pequeño productor y GP: Grande productor

Fuente: Elaboración propia con referencias de los tablas 6, 7, 8 y figura 3.

Tabla 10. Venta al día por participante de la cadena de comercialización.

\begin{tabular}{|c|c|c|c|c|c|}
\hline Datos* & $\begin{array}{c}\text { Unidad } \\
\text { de } \\
\text { medida }\end{array}$ & $\begin{array}{c}\text { Volumen de } \\
\text { venta (lt/día) }\end{array}$ & $\begin{array}{l}\mathrm{N}^{\mathrm{o}} \text { partici- } \\
\text { pantes }\end{array}$ & $\begin{array}{l}\text { Promedi } \\
\text { por par } \\
\text { (lt) }\end{array}$ & $\begin{array}{l}\text { de venta } \\
\text { cipante } \\
(\mathrm{kg})\end{array}$ \\
\hline Pequeño productor & Lt/día & $1,344.26$ & 86 & 15.63 & \\
\hline Mediano productor & $\mathrm{Lt} /$ día & $1,962.68$ & 45 & 43.62 & \\
\hline Grande productor & $\mathrm{Lt} /$ día & $1,495.55$ & 15 & 99.70 & \\
\hline Acopiador rural & $\mathrm{Lt} /$ día & $4,380.00$ & 6 & 730.00 & \\
\hline Procesador formal & $\mathrm{Kg}$ /día & 775.52 & 6 & 961.64 & 129.25 \\
\hline Procesador informal & Kg/día & $1,192.26$ & 14 & 573.99 & 85.16 \\
\hline Mercado Minorista de Lima & $\mathrm{Kg}$ /día & & 30 & 762.18 & 107.50 \\
\hline Mercado Modelo Huancayo & Kg/día & & 15 & 506.44 & 71.43 \\
\hline Mercado Modelo Huancayo & $\mathrm{Lt} /$ día & & 8 & 200.00 & \\
\hline \multicolumn{6}{|c|}{$\begin{array}{l}\text { Polumen de ventas y numero de participantes en productores, acopiadores y procesadores son obtenidos de la muestra } \\
\text { de queso o leche fresca. }\end{array}$} \\
\hline Elabo & & & & & \\
\hline
\end{tabular}


Tabla 11. Rentabilidad de la cadena de comercialización.

\begin{tabular}{|c|c|c|c|c|c|c|}
\hline Canal & $\begin{array}{c}\operatorname{Re}(\mathrm{S} / . / \\
\mathrm{mes})\end{array}$ & $\begin{array}{l}\text { Pequeño } \\
\text { productor } \\
\text { ganadero }\end{array}$ & $\begin{array}{l}\text { Acopiador } \\
\text { rural }\end{array}$ & $\begin{array}{c}\text { Planta } \\
\text { procesadora }\end{array}$ & $\begin{array}{c}\text { Minorista } \\
\text { (Lima) }\end{array}$ & $\begin{array}{l}\text { Modelo } \\
\text { (Huancayo) }\end{array}$ \\
\hline \multirow{2}{*}{3} & $\mathrm{P}$ & $\mathrm{S} / .84$ & $\mathrm{~S} / .2 .975$ & $\mathrm{~S} / .8 .508$ & $\mathrm{~S} / .2 .458$ & \\
\hline & G & S/. 313 & $\mathrm{~S} / .4 .946$ & $\mathrm{~S} / .8 .508$ & $\mathrm{~S} / .2 .458$ & \\
\hline \multirow{2}{*}{4} & $\mathrm{P}$ & $\mathrm{S} / .84$ & & $\mathrm{~S} / .13 .412$ & $\mathrm{~S} / .2 .458$ & \\
\hline & G & S/. 313 & & S/. 16.009 & $\mathrm{~S} / .2 .458$ & \\
\hline \multirow{2}{*}{6} & $\mathrm{P}$ & $\mathrm{S} / .84$ & $\mathrm{~S} / .2 .975$ & $\mathrm{~S} / .8 .508$ & & $\mathrm{~S} / .1 .054$ \\
\hline & G & S/. 313 & $\mathrm{~S} / .4 .946$ & $\mathrm{~S} / .8 .508$ & & $\mathrm{~S} / .1 .054$ \\
\hline \multirow{2}{*}{8} & $\mathrm{P}$ & S/. 84 & & S/. 2.976 & S/. 3.329 & \\
\hline & $\mathrm{G}$ & $\mathrm{S} / .313$ & & $\mathrm{~S} / .4 .526$ & $\mathrm{~S} / .3 .329$ & \\
\hline \multirow{2}{*}{9} & $\mathrm{P}$ & $\mathrm{S} / .84$ & $\mathrm{~S} / .2 .695$ & $\mathrm{~S} / .49$ & $\mathrm{~S} / .3 .329$ & \\
\hline & G & S/. 313 & S/. 4.946 & S/. 49 & $\mathrm{~S} / .3 .329$ & \\
\hline \multirow{2}{*}{10} & $\mathrm{P}$ & $\mathrm{S} / .84$ & & S/. 2.976 & & S/. 3.776 \\
\hline & G & $\mathrm{S} / .313$ & & $\mathrm{~S} / .4 .526$ & & S/. 3.776 \\
\hline \multirow{2}{*}{$12 \mathrm{~A}$} & $\mathrm{P}$ & $\mathrm{S} / .84$ & S/. 2.975 & & & S/. 299 \\
\hline & G & $\mathrm{S} / .313$ & $\mathrm{~S} / .4 .946$ & & & S/. 299 \\
\hline \multirow{2}{*}{$12 \mathrm{~B}$} & $\mathrm{P}$ & S/. 84 & & & & S/. 1.319 \\
\hline & $\mathrm{G}$ & S/. 313 & & & & S/. 1.859 \\
\hline
\end{tabular}

\section{Conclusiones}

En el Valle del Mantaro las interrelaciones entre agentes del sistema de producción y comercialización son asimétricas pues aquellos vinculados directamente a la transformación y el comercio poseen mayor conocimiento técnico y del mercado, en tanto los pequeños ganaderos toman decisiones con poca información, siendo que, la articulación con los proveedores de insumos productivos y servicios técnicos establecen diferencias tecnológicas entre productores grandes y pequeños, igualmente, las plantas procesadoras son distintas en la escala y la tecnología, siendo que pocas de ellas reúnen condiciones óptimas y existen transformadores informales en importante número que crean desorden y dificultan la organización del sistema.

En el mercado de quesos ocurre una oferta dual en la cadena de suministro, pues coexisten simultáneamente mercados formales e informales que impactan en los productores e intermediarios. Los supermercados demandan mayor calidad para consumidores de mayor nivel económico que son abastecidos por la industria formal, en tanto, los demandantes de menor ingreso son atendidos por transformadores artesanales, como Aubron (2006) señala "el queso artesanal es un producto para los pobres" dado su menor precio.

La comercialización de leche en el mercado rural es ineficiente dado: La red de comercialización centralizada por las plantas procesadoras; las fallas en la información; la carencia de contratos formales y de estandarización de la leche fresca, definiendo la venta a quién pague el mejor precio ese día; la desigual distribución de las ganancias unitarias; las dificultades a la libre entrada y salida del productor debido a que la reconversión productiva no es inmediata; la estacionalidad que genera exceso de oferta en la época de lluvias contrayendo los precios y en los meses de seca se elevan los precios; la existencia de mercados formales e informales en importante cuantía en el mismo territorio; la falta de registros para evaluar costos; la diversificación del productor pues las fuentes de ingresos incluyen cultivos agrícolas, crianza de animales menores y venta de mano de obra en el mercado regional. A pesar de los ratios, la actividad ganadera genera valor, ocupa la mano de obra local, utiliza los recursos productivos e incrementa los activos, su mejora debe contemplar el fortalecimiento de las asociaciones en torno a la producción y comercialización, pues sin ello será difícil reconocer sus propias deficiencias.

\section{Agradecimientos}

El estudio se desarrolló en el proyecto "Estrategias para mitigar el cambio climático y económico de la cadena productiva de la leche en el Valle del Mantaro, Junín, Perú" en alianza entre la Universidad Nacional Agraria La Molina y el Centro Francés de Cooperación Internacional en la Investigación Agrícola para el Desarrollo, siendo financiada por el Banco Mundial. Los autores agradecen a esas instituciones por su contribución.

\section{Literaura citada}

Alarcón, J. y Ordinola, M. 2002. Mercadeo de Productos Agropecuarios: Teoría y aplicaciones al Caso Peruano. (1 ${ }^{\text {ra }}$ Ed.). Perú: UNALM.

Álvarez, A. y Sánchez, B. 1998. Costos y Métodos de Costeo. Aplicación y análisis para el sector agropecuario. Universidad Nacional de Colombia. Medellín - Colombia.

Aubron, C. 2006. Productores andinos de queso artesanal y liberalización del mercado de lácteos en el Perú. En Debate Agrario 40-41, CEPES, Lima. 
Bienz, N. 2011. Cultivating Prospective thinking: A Gateway into the Future for Peruvian Dairy Farmers in the Mantaro Valley. Experimenting a Support Approach Based on the use of Modeling Tools. MSc Thesis Sustainable Development in Agriculture. UMR Innovation - CIRAD

Caldentey, P. y Gómez, A. 1992. Economía de los mercados agrarios. Madrid: Universidad de Córdoba.

Cannock y Gonzáles-Zuñiga 1994, Economía Agraria, Universidad del Pacífico. Lima - Perú.

Cortijo, E.; Faure, G. y Le Gal, P. 2010. Inserción de las pequeñas explotaciones familiares en la cadena de suministro de los lácteos en el Valle del Mantaro (Perú): hacia una gestión de apoyo que tome en cuenta la diversidad de los actores. Banco Mundial - Cirad n ${ }^{\circ}$ 7151108 .

Coscia A. (1978), Comercialización de Productos Agropecuarios. Argentina.

Espinoza, V.; Rivera, G. y Garcia, L. 2008 Los canales y márgenes de comercialización de la leche cruda producida en sistema familiar (estudio de caso). Vet. Méx [online]. vol.39, n.1, pp. 1-16. ISSN 0301-5092

Gave A. 2010. Caracterización de la actividad lechera en las asociaciones de productores ganaderas de la provincia de Jauja región Junín. Tesis Ingeniero Zootecnista, Huancayo - Perú: Universidad Nacional del centro del Perú.

Heady, P. 2005. Barter, in A Handbook of Economic Anthropology. Published by Edward Elgar Publishing. UK. 16: 263.

Huamanchaqui, J. y Porras L. 2004. Caracterización de la producción y comercialización de la leche fresca en el valle del Mantaro. Tesis Ingeniero Zootecnista, Huancayo - Perú: Universidad Nacional del centro del Perú.

Martínez, F. 2005. Comercialización Agropecuaria: Un enfoque económico de las estrategias comerciales. (1 $1^{\text {ra }}$ Ed.). Chile: Pontificia Universidad Católica de Chile

Mayer, E. y Glave, M. 1999. A little something to earn: profit and losses in peasant economies. American Ethnologist 26: 344-69.

Mayer, E. y Glave, M. 1999. A little something to earn: profit and losses in peasant economies. American Ethnologist 26: 344-69.

Meléndez, R 1984. Mercadeo de Productos Agropecuarios. México.

Mendoza, G. 1987. Compendio de Mercadeo de Productos Agropecuarios ( $2^{\text {da }}$ Ed.). Costa Rica: IICA

Ministerio de Agricultura, Dirección Regional de Agricultura de Junín 2006. División Política y Organización Administrativa.

Ortiz, S. 2005. Decisions and choices: the rationality of economic actors, in A Handbook of Economic Anthropology. Published by Edward Elgar Publishing. UK. 4: 67.
(Footnotes)1La interrelación entre ganadero y proveedores de bienes y servicios se mide en porcentaje de productores que acceden a ellos. 